\title{
Competencias digitales en la formación de tutores virtuales en la Universidad Técnica del Norte, Ibarra-Ecuador
}

\author{
Andrea V. Basantes-Andrade ${ }^{1}$, Marcos Cabezas-González ${ }^{2}$ y Sonia Casillas-Martín ${ }^{2}$ \\ (1) Universidad Técnica del Norte, Grupo de Investigación de Ciencias en Red (e-CIER), Av. 17 de Julio 5-21 y General \\ José María Córdova, Código postal 100105, Ibarra - Ecuador. (correo-e: avbasantes@utn.edu.ec) \\ (2) Universidad de Salamanca, Paseo de Canalejas 169, Salamanca-España. (correo-e: mcabezasgo@usal.es; \\ scasillasma@usal.es)
}

Recibido Mar. 24, 2020; Aceptado May. 25, 2020; Versión final Jun. 11, 2020; Publicado Oct. 2020

\begin{abstract}
Resumen
En este artículo se presenta un estudio de caso que identifica y ratifica la importancia de las competencias digitales en la formación de los docentes relacionados con la educación virtual. Se describe el diseño e implementación de un curso online, basado en la metodología PACIE, el cual estuvo orientado a identificar y desarrollar las competencias digitales de 208 futuros tutores virtuales. La investigación se realizó bajo un enfoque mixto (cuali-cuantitativo) que evidenció el valor de dichas competencias para que los tutores puedan apoyar y fortalecer los procesos pedagógicos y formativos de los estudiantes dentro del aula virtual. El $87 \%$ de los encuestados afirman haber mejorado dichas competencias durante el desarrollo del curso en línea. En conclusión, la formación de tutores virtuales en competencias digitales es esencial para desarrollar el pensamiento crítico, habilidades, conocimientos, actitudes y estrategias de uso eficiente y seguro de las tecnologías de información y comunicación en la educación en línea.
\end{abstract}

Palabras clave: competencias digitales; formación docente; educación en línea; TIC; metodología PACIE

\section{Digital competencies in the training of virtual tutors at the Universidad Técnica del Norte, Ibarra (Ecuador)}

\begin{abstract}
This paper presents a case study that identifies the importance of digital competencies in virtual education tutor training. This research study describes the design and implementation of an online course based on the PACIE methodology, which was aimed at identifying and developing e-skills of 208 virtual tutor trainees. The research was performed under a mixed approach (qualitative-quantitative). The results show the value of eskills required by tutors to support and strengthen the pedagogical and formative processes of students within virtual classrooms. Most surveyed trainees claim to have improved their e-skills during the course (87\%). In conclusion, training of virtual tutors in digital skills is essential to develop critical thinking, skills, knowledge, attitudes, and strategies for the efficient and safe use of information and communication technologies in online education.
\end{abstract}




\section{INTRODUCCIÓN}

El objetivo principal de esta investigación estuvo en identificar y desarrollar las competencias digitales de 208 futuros tutores virtuales. El artículo se divide en cinco secciones: primero, una introducción donde se detalla el problema y los objetivos de la investigación; segundo, metodología, describe la organización, los métodos y las tareas llevadas a cabo durante el curso virtual; tercero, los resultados, muestra los datos obtenidos en las encuestas realizadas a los participantes; cuarto, discusión, se explica los resultados y se compara los mismo con los hallazgos de otros investigadores; y finalmente, conclusiones tras analizar los resultados de la investigación y realizar la discusión se estable las conclusiones de este estudio.

Las universidades se configuran como un elemento estructural de desarrollo del conocimiento, encargadas de la formación del capital humano como uno de los principales actores de cambio para la sociedad contemporánea. Una de las disrupciones más importantes suscitadas en los últimos años dentro del ámbito educativo es el aprendizaje en línea, más conocido como e-learning, este sistema se basa en el uso de tecnologías y recursos digitales para crear un entorno de aprendizaje personalizado, interactivo, colaborativo y fundamentalmente flexible en tiempo y espacio (Rodrigues et al., 2019). Este entorno virtual hace que el estudiante sea el protagonista del proceso de enseñanza-aprendizaje, anexando un alto componente de auto-criticidad y responsabilidad (Basantes et al., 2017). Sin embargo, la metodología de enseñanza-aprendizaje que utiliza el docente parece desvinculada de la realidad, disuade el desarrollo del aprendizaje, así como la adquisición de las habilidades o competencias necesarias para desenvolverse en el siglo XXI.

Consecuentemente, la educación debe ir en la misma dirección que los procesos de transformación socio cultural que tienen su punto de partida en los métodos, competencias y herramientas generados alrededor de las Tecnologías de Información y Comunicación (TIC) (Villarreal-Villa et al., 2019). Las competencias digitales, también llamadas e-skills, hacen referencia al conjunto de conocimientos y habilidades que posibilitan el uso seguro y eficiente de las Tecnologías de Información y Comunicación (Janssen et al., 2013); estas competencias en educación virtual toman especial relevancia debido a la pérdida de la interacción física entre alumno y tutor, es imprescindible el uso de métodos alternativos para superar estos obstáculos y promover el aprendizaje. Los tutores de educación en línea deben orientar sus capacidades, valores, estrategias y actitudes es pos de generar una comunicación efectiva, un intercambio de información con mayor fluidez y sobre todo una inmersión pedagógica que permita evaluar y examinar las etapas del proceso de eneseñanza-aprendizaje de acuerdo al entorno que se utilice para su desarrollo (Martínez et al., 2018). Según Røkenes-Krumsvik (2016) y Lorente (2006) las competencias básicas que un tutor virtual debe poseer son: académica, organizativa, orientadora, técnica y social. Ver Tabla 1.

Tabla 1: Competencias básicas del tutor virtual

\begin{tabular}{|l|l|}
\hline $\begin{array}{l}\text { Tipos de } \\
\text { competencias }\end{array}$ & Detalle \\
\hline Académicas & $\begin{array}{l}\text { Investigar y profundizar temas } \\
\text { Estructurar el conocimiento } \\
\text { Informar, clarificar y explicar los contenidos } \\
\text { Diseñar actividades y situaciones de aprendizaje (individual y grupal) } \\
\text { Resolver dudas surgidas durante el desarrollo de las actividades } \\
\text { Promover el trabajo colaborativo } \\
\text { Crear interrogantes para generar debate y propiciar la interacción } \\
\text { Colegir o sintetizar los debates (individuales o grupales) }\end{array}$ \\
& $\begin{array}{l}\text { Asignar y realizar valoraciones globales e individuales de las actividades. } \\
\text { Evaluar y retroalimentar el aprendizaje }\end{array}$ \\
\hline Organizativas & $\begin{array}{l}\text { Explicar las normas y funcionamiento del entorno virtual } \\
\text { Establecer fechas y horarios para la participación en foros y chats } \\
\text { Mantener contacto con el resto del equipo docente y organizativo } \\
\text { Organizar el trabajo en grupo y facilitar la coordinación entre los miembros } \\
\text { Establecer los canales de comunicación online y sus directrices } \\
\text { Organizar las tareas administrativas }\end{array}$ \\
\hline Orientadoras & $\begin{array}{l}\text { Facilitar técnicas de trabajo intelectual para el estudio en red } \\
\text { Dar recomendaciones para mejorar el rendimiento y la calidad del trabajo académico } \\
\text { Incentivar el trabajo en red } \\
\text { Informar a los estudiantes sobre su progreso en el estudio } \\
\text { Ser guía y orientador del estudiante }\end{array}$ \\
\hline
\end{tabular}


Tabla 1: continuación.

\begin{tabular}{|c|c|}
\hline Técnicas & $\begin{array}{l}\text { Asegurarse de que los alumnos comprenden el funcionamiento técnico del entorno telemático de formación } \\
\text { Conocer la plataforma virtual y los servicios que incorpora para el seguimiento y acompañamiento del } \\
\text { aprendizaje del estudiante } \\
\text { Gestionar los grupos de aprendizaje para el trabajo en la red } \\
\text { Incorporar y modificar nuevos materiales al entorno formativo } \\
\text { Mantenerse en contacto con el administrador del sistema } \\
\text { Utilizar adecuadamente el correo electrónico } \\
\text { Saber dirigir y participar en comunicaciones asincrónicas y síncronas }\end{array}$ \\
\hline Sociales & $\begin{array}{l}\text { Dar la bienvenida a los estudiantes que participan en el curso } \\
\text { Incitar a los estudiantes para que amplíen y desarrollen los argumentos presentados por sus compañeros } \\
\text { Moderar las discusiones; es decir, integrar y conducir las intervenciones } \\
\text { Animar y estimular la participación } \\
\text { Proponer actividades para facilitar el conocimiento entre los participantes } \\
\text { Dinamizar la acción formativa y el trabajo en red } \\
\text { Realizar el seguimiento personalizado a cada estudiante }\end{array}$ \\
\hline
\end{tabular}

Para las Instituciones de Educación Superior (IES), esta modalidad de estudio demanda la implementación de una estructura, tanto organizacional como tecnológica, que permita alcanzar una formación humana de calidad, misma que contribuya a maximizar el potencial de los estudiantes (McDougall, 2019). Dentro de este contexto, las competencias digitales cobran cada vez mayor importancia en los procesos de enseñanza-aprendizaje, debido principalmente al vertiginoso desarrollo de las Tecnologías de la Información y Comunicación (TIC) y el Internet (Nyikes, 2018). Autores como Pérez y Rodríguez (2016), Tømte et al., (2015) señalan que, la formación en competencias digitales es necesaria para todos los docentes, a fin de que su participación en la sociedad digital sea adecuada, capacitándolos para transferir sus conocimientos y habilidades dentro de los entornos virtuales de aprendizaje, caracterizados por su flexibilidad configurativa y ubicuidad.

No es suficiente realizar acciones formativas sobre el uso de la tecnología para lograr una adopción efectiva de las TIC en el aula, según Cabero y Martínez (2019) la formación docente debe basarse en tres principios: 1) abordar la formación desde un enfoque pedagógico que permita la adaptación y apropiación de la tecnología; es decir, enfocar el uso de metodologías activas respaldadas por las TIC como el aprendizaje basado en proyectos, aprendizaje basado en problemas, estudios de casos, aula invertida entre otros (Garzón et al., 2020); 2) es necesario abordar la formación desde diferentes enfoques culturales, sociales, pragmáticos, psicológicos, cognitivos, curriculares, tecnológicos, comunicativos u otros; y 3) vislumbrar diversos principios y valores éticos, reflexionar sobre su nivel de competencia digital, desarrollar el pensamiento crítico, establecer estrategias de formación basadas en la colaboración e interacción social, resolución de casos prácticos y reales de acuerdo al contexto, desarrollar la comunicación efectiva y asertiva entre otros aspectos. A estos tres principios se añade el diagnóstico previo antes de iniciar la formación con la finalidad de construir un plan de formación adaptado a las necesidades formativas del docente.

Cuanto mayor sea el compromiso del docente para actualizar su competencia digital, mayor será su proyección formativa, enriquece su base de conocimientos, habilidades y destrezas, métodos de enseñanza, herramientas digitales, destinados a comprender y mejorar la formación de una nueva generación de estudiantes, que nace y crece en la era digital (Basantes-Andrade et al., 2020). A nivel internacional se identifican varios modelos y marcos conceptuales que intentan caracterizar la competencia digital docente (Caena et al., 2019; INTEF, 2017; Prendes et al., 2018), estos no especifican los estándares de competencia para la formación de tutores virtuales; sin embargo, han aportado una serie de ideas relevantes para establecer un plan integral de capacitación en esta área. Esta formación debe estar articulada con las políticas del Estado y las Instituciones de Educación Superior.

En el Ecuador, la Constitución de la República tipifica a la educación de tercer nivel como un derecho que debe ser garantizado por el Estado, sin embargo, la oferta académica existente no cubre las demandas de ingreso de nuevos estudiantes que anualmente se suscita. Es por esta razón que, en el marco de una estrategia integral para incrementar la oferta académica y democratizar el acceso a la educación superior (Senescyt, 2018), la Secretaría de Educación Superior, Ciencia, Tecnología e Innovación impulsó un Programa de Educación Superior Virtual, mediante un trabajo conjunto con cinco universidades públicas del país: Universidad Técnica del Norte (Ibarra), Universidad Central del Ecuador (Quito), Universidad Estatal de Milagro (Milagro), Universidad Técnica de Manabí (Portoviejo) y la Escuela Politécnica del Ejército (Sangolquí). Estudios relacionados con la formación de competencias digitales coligen que, la competencia digital de los docentes en Ecuador es baja (Cazco et al., 2016), lo que está relacionado directamente con los problemas de aprendizaje de los estudiantes, en especial, para los que optan por una modalidad de estudio virtual. 


\section{OTROS ANTECEDENTES}

Para dar solución a esta problemática, la Universidad Técnica del Norte (UTN) estableció como prioridad dentro de sus líneas estratégicas, el fortalecimiento de las competencias digitales de todos sus docentes, haciendo énfasis sobre aquellos responsables de llevar a cabo su oferta académica virtual. Se desarrolló un curso online orientado a identificar y mejorar las competencias digitales de 208 docentes universitarios relacionados con la educación virtual. En consideración, de que la UTN utilizó la metodología PACIE como diseño instruccional para crear el Campus Educativo Virtual y esta forma parte del modelo educativo de la institución, se eligió la misma metodología para crear el curso en línea, el cual se implementó en la plataforma Moodle.

PACIE, es el resultado de sus cinco fases secuenciales del diseño instruccional: $(P)$ presencia, representa el desarrollo de la imagen corporativa, interfaz y navegabilidad del Entorno Virtual de Aprendizaje (EVA); (A) alcance, se define el fin pedagógico y los objetivos que se desea alcanzar; (C) capacitación, actualización oportuna acorde a las necesidades formativas; (I) interacción, selección de los recursos y actividades que permite generar la participación social y la co-creación del conocimiento y (E) e-learning), es la puesta en marcha del proceso de formación en línea (Basantes-Andrade et al., 2019).Esta metodología integra una estrategia tecno-pedagógica para administrar, gestionar, monitorear y evaluar las actividades y tareas realizadas por los asistentes al curso; con ello, la formación docente no se centra en la instrumentalización de la tecnología digital en el aula sino en las habilidades de comprensión tecno pedagógicas necesarias para adaptarse a los escenarios educativo reales y posibilitar que los estudiantes den el salto de la Sociedad de la Información a la Sociedad del Conocimiento.

Los entornos virtuales de aprendizaje precisan de una metodología que, por medio de la integración tecnopedagógica de las TIC permita optimizar los procesos de enseñanza-aprendizaje virtual. La metodología PACIE en los Entornos Virtuales de Aprendizaje (EVA) contribuye a alcanzar los objetivos en el proceso de enseñanza-aprendizaje, a través de la integración de las TIC y bajo un enfoque pedagógico, constructivista, colaborativo y fundamentalmente basado en la sinergia entre profesor y alumno (Basantes et al., 2018). La estructura interna de un curso virtual bajo la metodología PACIE ha sido probada en aulas de programas de experto por la Fundación para la Actualización Tecnológica de Latinoamérica (FATLA), esta estructura se divide en tres macro-bloques: 1) bloque 0 (cero) o PACIE, organiza y propicia la gestión e interacción en el aula virtual, 2) bloque académico, aloja los contenidos temáticos, actividades y evaluación del aprendizaje, pasando de lo conceptual a la praxis; y 3) bloque de cierre, posibilita la finalización de los procesos que han quedado inconclusos dentro del curso o de la labor tutorial. Cada uno de los bloques se subdivide en múltiples secciones, las cuales se encuentran definidas y organizadas como se puede observar en la Figura 1.

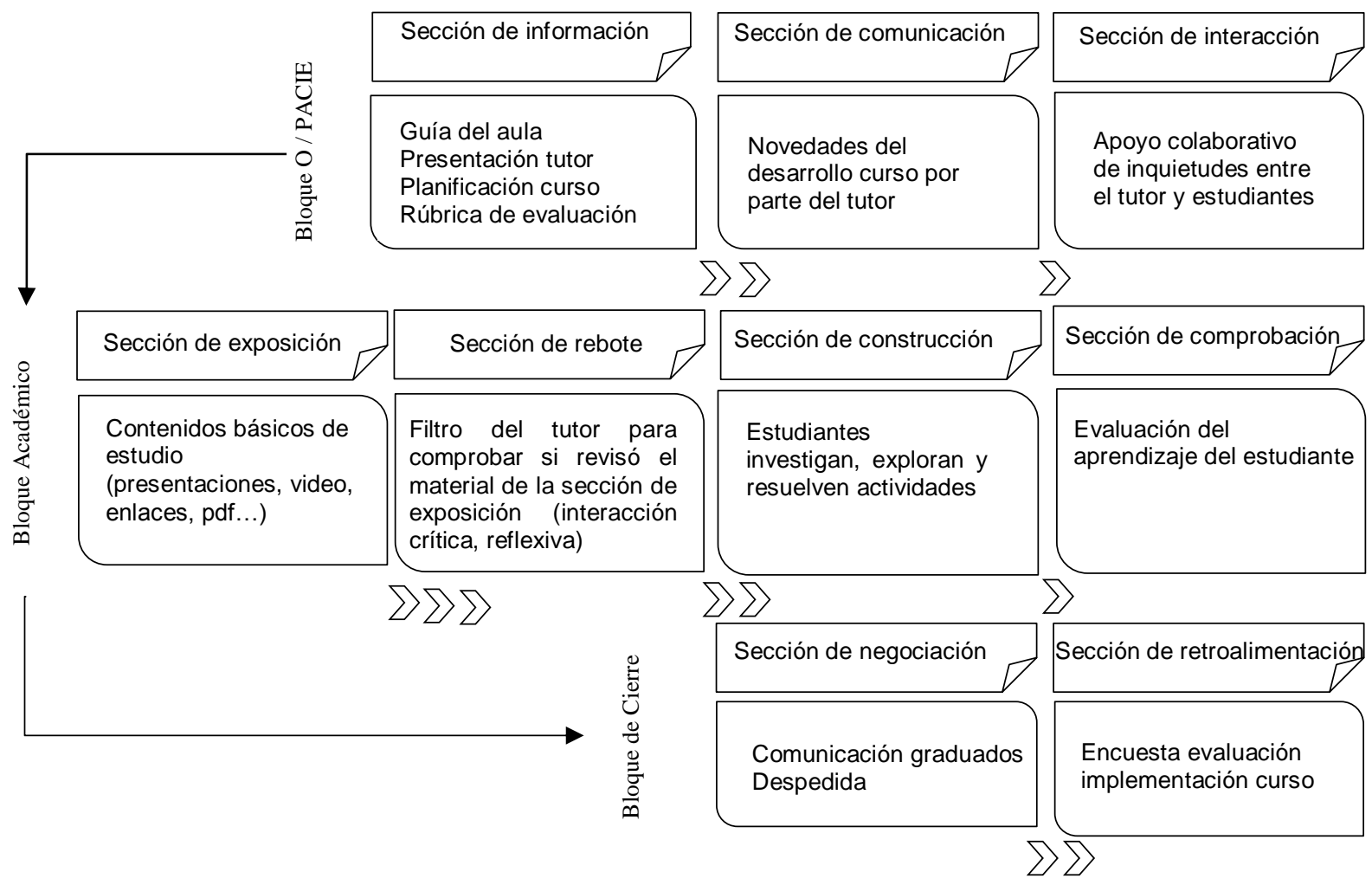

Fig. 1: Estructura conceptual de la metodología PACIE 


\section{METODOLOGÍA}

Esta investigación se realizó bajo un enfoque mixto (cualitativo-cuantitativo), con un estudio de caso que se dividió en tres fases: revisión bibliográfica, diseño de la investigación, y desarrollo y aplicación

\section{Fase 1. Revisión bibliográfica}

Con la finalidad de establecer los contenidos, alcance y actividades del curso para la formación de tutores virtuales (online), fue indispensable realizar una exhaustiva revisión bibliográfica, a fin de identificar, evaluar e interpretar las investigaciones relevantes sobre competencias digitales que un tutor virtual debe poseer para apoyar y facilitar los procesos de aprendizaje en línea de los estudiantes. La revisión se realizó a través de los artículos publicados en diferentes bases de datos científicas como Web of Sciense, Scopus y Scielo, y las bibliotecas virtuales de la Universidad de Salamanca (España) y la Universidad Técnica del Norte (Ibarra-Ecuador). Se utilizó el gestor bibliográfico Mendeley para buscar, almacenar, organizar y analizar las referencias y notas bibliográficas consultadas. Los términos o palabras claves utilizadas para la búsqueda de la información fueron: competencias digitales, alfabetización digital, formación digital, habilidades de internet y recursos tecnológicos educativos

Una vez extraída la información se procedió a dar respuesta a la interrogante primaria de esta investigación: ¿Cuáles son las competencias digitales básicas que un tutor virtual debe poseer? La mayoría de los autores hacen referencia al Marco Común de Competencias Digitales Docentes (INTEF, 2017) que implica el uso crítico y seguro de las TIC para el trabajo, ocio y la comunicación. En base a estas referencias, se esquematizó una descripción de la literatura utilizada con relación a los cinco pilares de las competencias digitales: Información y alfabetización informacional, Comunicación y colaboración, Creación de contenido digital, Seguridad y Resolución de problemas. En la Figura 2 se muestra las cinco áreas competenciales que un tutor virtual debe poseer, con una descripción de cada uno de ellos.

\section{COMPETENCIAS DIGITALES DE UN TUTOR VIRTUAL}

01

INFORMACIÓN Y ALFABETIZACIÓN INFORMACIONAL

Busca, identifica, almacena, organiza y analiza la información relevante.

Maneja eficientemente el entorno de la plataforma en línea.

Identifica los recursos y actividades de la plataforma en línea.

Actualiza su conocimiento para emplear nuevas estrategias metodológicas con TIC.

Aplica metodologías docentes emergentes.

02 COMUNICACIÓN Y COLABORACIÓN

Aplica herramientas TIC para la comunicación síncrona y asíncrona.

Planifica, ejecuta y evalúa una videoconferencia para gestionar actividades académicas (docencia, investigación, gestión y vinculación).

Proporciona lineamientos de cómo usar los medios y recurso de forma efectiva.

Promueve la participación e interacción social basado en la reflexión y crítica constructiva.

- Establece las claves para realizar una presentación eficaz (oral o escrita).

03 CREACIÓN DE CONTENIDO DIGITAL

Maneja herramientas TIC que pueden impulsar la innovación docente en el aula virtual.

Diseña estrategias para facilitar el aprendizaje.

- Analiza y emplea herramientas TIC de acuerdo con las características de los estudiantes y el objetivo que desea alcanzar.

- Selecciona las TIC que promueven el desarrollo de habilidades digitales.

- Establece criterios para evaluar el desempeño formativo, sumativo del estudiante.

- Especifica el plan de retroalimentación (feedback) del aprendizaje de los estudiantes.

\section{SEGURIDAD}

Respeta los derechos de autor de recursos utilizados.

Establece el uso correcto de las herramientas TIC como instrumento de apoyo para la planificación, ejecución y evaluación de la enseñanza-aprendizaje.

- Utiliza herramientas y mecanismos para el seguimiento y acompañamiento del aprendizaje del estudiante.

05 RESOLUCIÓN DE PROBLEMAS

Conoce las estrategias, técnicas y medios tecnológicos para controlar situaciones problemáticas.

Fig. 2: Competencias digitales de un tutor virtual. Adaptado de INTEF, 2017 


\section{Fase 2. Diseño de la investigación}

La población o universo para el desarrollo de la investigación estuvo conformado por 208 personas $(65,87 \%$ hombres y $43,13 \%$ mujeres), las cuales, se inscribieron en el curso online para la "Formación de Tutores Virtuales", ofertado por la Empresa Pública "La Uemprende EP" de la Universidad Técnica del Norte. No se realizó ningún cálculo muestral y se trabajó con toda la población existente. Es preciso señalar que el número total de docentes en la UTN es de 588 , de los cuales el $35 \%$ se interesó en el curso y en la mejora de sus competencias digitales.

Antes de iniciar el curso, se realizó un diagnóstico sobre las competencias digitales que cada uno de los participantes poseía; con este propósito, se utilizaron los formularios de Google para realizar la respectiva encuesta digital. Los datos obtenidos demostraron las falencias en competencias digitales de los participantes en el curso (ver Tabla 3). Tres expertos del área de Tecnología Educativa de la Universidad Técnica del Norte validaron el instrumento, el cual, se conformó con 10 preguntas de selección múltiple. Para comprobar la consistencia interna de la encuesta, se utilizó Alfa de Cronbach, cuyo coeficiente alcanzó el valor de 0,89; valoración que corrobora la alta fiabilidad del instrumento. La tabulación de los datos y el análisis descriptivo de los mismos se realizó por medio del paquete estadístico SPSS v22.0.

Tabla 3: Resultados diagnóstico de la competencia digital en nivel avanzado

\begin{tabular}{|c|c|c|}
\hline Componente competencia digital & Resultado & Promedio competencia \\
\hline \multicolumn{2}{|l|}{ Información y alfabetización informacional } & \multirow[t]{3}{*}{$47.83 \%$} \\
\hline Almacenar la información en Google Drive, OneDrive, Dropbox & $54.32 \%$ & \\
\hline Buscar información en Internet usando estrategias de navegación & $41.34 \%$ & \\
\hline \multicolumn{2}{|l|}{ Comunicación y colaboración } & \multirow[t]{4}{*}{$42.54 \%$} \\
\hline Compartir información en la Red & $52.38 \%$ & \\
\hline Redes sociales Facebook, Twitter y YouTube & $32.70 \%$ & \\
\hline Zoom, Hangouts, Skype & $42.53 \%$ & \\
\hline \multicolumn{2}{|l|}{ Creación de Contenido Digital } & \multirow[t]{4}{*}{$24.67 \%$} \\
\hline Prezi, Emaze, Sway & $17.30 \%$ & \\
\hline Office 365 & $54.31 \%$ & \\
\hline Kahoot & $2.40 \%$ & \\
\hline \multicolumn{2}{|l|}{ Seguridad } & \multirow[t]{3}{*}{$2.92 \%$} \\
\hline Seguridad de los datos, netiqueta e identidad digital & $3.5 \%$ & \\
\hline Derechos de autor y licencias de uso & $2.33 \%$ & \\
\hline \multicolumn{2}{|l|}{ Resolución de problemas } & \multirow[t]{2}{*}{$49.03 \%$} \\
\hline Configuraciones básicas de dispositivos digitales & $49.03 \%$ & \\
\hline
\end{tabular}

\section{Fase 3. Desarrollo y Aplicación}

Con los datos obtenidos en las fases 1 y 2 , se dimensionaron y establecieron siete módulos en total para la formación de los tutores virtuales. En la Tabla 4 se presenta la estructura y organización de cada módulo en relación a los siguientes aspectos: 1) nivel de aprendizaje, se relaciona directamente con los objetivos de cada módulo, los mismos que constituyen el hilo conductor de la acción formativa, expresan de forma clara y concreta los propósitos (metas) que persigue el curso (¿qué van a aprender los estudiantes?), las condiciones y el tiempo en el que se esperan alcanzar los mismos. Para la redacción de los objetivos se basó en la taxonomía de Bloom, quien jerarquiza los procesos mentales cognitivos desde un nivel inferior (recordar y comprender), nivel intermedio (aplicar y analizar) y un nivel superior (evaluar y crear); 2) competencias; se hace referencia a las competencias digitales que se desarrollará en los participantes del curso; 3) contenidos, se procuró conservar las propiedades de la información que le permite al estudiante el poder asimilar, comprender y procesar la información recibida (Martínez et al., 2018); 4) estrategias didácticas, las que promueven la formación integral de los estudiantes abarcando los ámbitos cognitivo y afectivo a través de las interacciones sociales y la comunicación mediada por las TIC (Oses y Carrasco, 2013); y 4) recursos, necesarios para el perfeccionamiento tutorial, la construcción del conocimiento y la adquisición de competencias personales y/o profesionales. 
Tabla 4: Estructura del curso online

\begin{tabular}{|c|c|c|c|c|c|}
\hline Módulos & $\begin{array}{c}\text { Nivel de } \\
\text { aprendizaje }\end{array}$ & $\begin{array}{c}\text { Competencias } \\
\text { Digitales }\end{array}$ & $\begin{array}{l}\text { Descripción } \\
\text { contenidos }\end{array}$ & $\begin{array}{l}\text { Estrategias } \\
\text { didácticas }\end{array}$ & Recursos \\
\hline Inicio & Básico & $\begin{array}{l}\text { Información y } \\
\text { alfabetización } \\
\text { informacional } \\
\text { Comunicación } \\
\text { y colaboración }\end{array}$ & $\begin{array}{l}\text { Introducción al } \\
\text { entorno de la } \\
\text { plataforma } \\
\text { Moodle y sus } \\
\text { servicios a fin de } \\
\text { que el docente- } \\
\text { estudiante se } \\
\text { familiarice con la } \\
\text { misma y conozca } \\
\text { los medios de } \\
\text { comunicación que } \\
\text { se usarán para } \\
\text { solventar } \\
\text { cualquier duda o } \\
\text { informarse sobre } \\
\text { las novedades del } \\
\text { desarrollo del } \\
\text { curso. } \\
\text { Interacción social. }\end{array}$ & $\begin{array}{l}\text { Actualización del } \\
\text { perfil en el entorno } \\
\text { Presentaciones } \\
\text { participantes, video } \\
\text { análisis (salir de la } \\
\text { zona de confort) } \\
\text { Test de evaluación }\end{array}$ & $\begin{array}{l}\text { Video-tutorial } \\
\text { (Screemcast-O- } \\
\text { Matic) } \\
\text { Foro-video } \\
\text { (Flipgrid) } \\
\text { Plataforma } \\
\text { Moodle }\end{array}$ \\
\hline $\begin{array}{l}\text { Tutor virtual } \\
\text { en Entornos } \\
\text { Virtuales de } \\
\text { Aprendizaje }\end{array}$ & Intermedio & $\begin{array}{l}\text { Comunicación } \\
\text { y colaboración } \\
\text { Resolución de } \\
\text { problemas }\end{array}$ & $\begin{array}{l}\text { Características, } \\
\text { evolución, } \\
\text { factores y retos de } \\
\text { la educación } \\
\text { virtual, } \\
\text { metodología } \\
\text { PACIE, el rol y } \\
\text { las competencias } \\
\text { que el tutor virtual } \\
\text { debe desarrollar y } \\
\text { el rol del } \\
\text { estudiante virtual. }\end{array}$ & $\begin{array}{l}\text { Foro de discusión: } \\
\text { paradigmas de la } \\
\text { educación virtual } \\
\text { Taller evaluación } \\
\text { por pares: Matriz de } \\
\text { factores de la } \\
\text { educación virtual } \\
\text { Test de evaluación } \\
\text { Sesión académica } \\
\text { presencial virtual }\end{array}$ & $\begin{array}{l}\text { Foro (recurso } \\
\text { Moodle) } \\
\text { Plataforma } \\
\text { Moodle } \\
\text { Zoom }\end{array}$ \\
\hline $\begin{array}{l}\text { Procesos } \\
\text { Tutor Virtual }\end{array}$ & $\begin{array}{c}\text { Nivel } \\
\text { superior }\end{array}$ & $\begin{array}{l}\text { Información y } \\
\text { alfabetización } \\
\text { informacional } \\
\text { Comunicación } \\
\text { y colaboración } \\
\text { Creación de } \\
\text { contenidos } \\
\text { digitales } \\
\text { Seguridad }\end{array}$ & \begin{tabular}{l}
\multicolumn{3}{l}{ Comunicación, } \\
interacción social \\
y colaboración, la \\
evaluación del \\
proceso \\
aprendizaje, de \\
seguimiento y \\
acompañamiento \\
del profesor tutor \\
hacia \\
estudiante, el \\
herramientas del \\
profesor tutor y \\
metodologías \\
docentes \\
emergentes
\end{tabular} & $\begin{array}{l}\text { Foro de discusión: } \\
\text { acompañando al } \\
\text { estudiante } \\
\text { Tarea: presentación } \\
\text { a sus estudiantes } \\
\text { Estudio de casos: } \\
\text { qué y cómo puede } \\
\text { ayudar a sus } \\
\text { estudiantes con una } \\
\text { corrección. } \\
\text { Test de evaluación } \\
\text { Sesión académica } \\
\text { presencial virtual }\end{array}$ & $\begin{array}{l}\text { Presentación } \\
\text { (Sway u otro) } \\
\text { Documento } \\
\text { Plataforma } \\
\text { Moodle } \\
\text { Zoom }\end{array}$ \\
\hline $\begin{array}{l}\text { Herramientas } \\
\text { TIC en el aula }\end{array}$ & $\begin{array}{c}\text { Nivel } \\
\text { superior }\end{array}$ & $\begin{array}{c}\text { Información y } \\
\text { alfabetización } \\
\text { informacional } \\
\text { Comunicación } \\
\text { y colaboración } \\
\text { Creación de } \\
\text { contenidos } \\
\text { digitales } \\
\text { Seguridad } \\
\text { Resolución de } \\
\text { problemas }\end{array}$ & 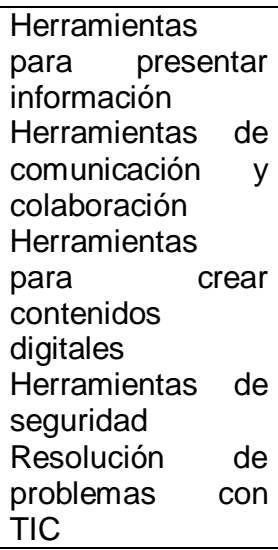 & $\begin{array}{l}\text { Glosario } \\
\text { colaborativo de } \\
\text { herramientas TIC } \\
\text { en el aula } \\
\text { Trabajo } \\
\text { colaborativo: } \\
\text { planificación y } \\
\text { desarrollo } \\
\text { videoconferencia } \\
\text { Test de evaluación } \\
\text { Sesión académica } \\
\text { presencial virtual }\end{array}$ & $\begin{array}{l}\text { Presentación } \\
\text { online (Emaze, } \\
\text { Visme, } \\
\text { Symbaloo, } \\
\text { Powtoon) } \\
\text { Videoconferenci } \\
\text { a (Zoom, } \\
\text { Hangouts u otro) } \\
\text { Plataforma } \\
\text { Moodle } \\
\text { Zoom }\end{array}$ \\
\hline
\end{tabular}


Tabla 4: continuación.

\begin{tabular}{|c|c|c|c|c|c|}
\hline $\begin{array}{c}\text { Objetos } \\
\text { Virtuales de } \\
\text { Aprendizaje } \\
\text { (OVA) }\end{array}$ & $\begin{array}{c}\text { Nivel } \\
\text { superior }\end{array}$ & $\begin{array}{l}\text { Creación de } \\
\text { Contenido } \\
\text { Digital } \\
\text { Seguridad } \\
\text { Resolución de } \\
\text { problemas }\end{array}$ & $\begin{array}{l}\text { Diseño instruccional } \\
\text { de Objetos Virtuales } \\
\text { de Aprendizaje, } \\
\text { planificación, díselo, } \\
\text { secuenciación y } \\
\text { elaboración de } \\
\text { OVA, derechos de } \\
\text { autor y licencias de } \\
\text { uso de recursos, y } \\
\text { Scorm }\end{array}$ & $\begin{array}{l}\text { Foro de discusión: } \\
\text { modelos de diseño } \\
\text { instruccional } \\
\text { Aprendizaje basado } \\
\text { en problemas: } \\
\text { Objeto Virtual de } \\
\text { Aprendizaje en } \\
\text { Scorm } \\
\text { Sesión académica } \\
\text { presencial virtual }\end{array}$ & $\begin{array}{l}\text { ExeLearning, } \\
\text { Constructor } \\
\text { Plataforma } \\
\text { Moodle } \\
\text { Zoom }\end{array}$ \\
\hline $\begin{array}{l}\text { Examen } \\
\text { presencial }\end{array}$ & & & $\begin{array}{lr}\text { Se realiza un } \\
\text { examen general de } \\
\text { todos } & \text { los } \\
\text { contenidos en los } \\
\text { módulos a fin de } \\
\text { evaluar } & \text { el } \\
\text { aprendizaje de los } \\
\text { mismos } & \\
\end{array}$ & $\begin{array}{l}\text { Examen integral } \\
\text { presencial } \\
\text { (evaluación } \\
\text { sumativa) }\end{array}$ & $\begin{array}{l}\text { Plataforma } \\
\text { Moodle }\end{array}$ \\
\hline Graduación & & & $\begin{array}{l}\text { Se proporciona la } \\
\text { certificación de } \\
\text { aprobación del } \\
\text { curso si completó y } \\
\text { cumplió con todas } \\
\text { las actividades } \\
\text { establecidas en el } \\
\text { curso }\end{array}$ & $\begin{array}{l}\text { Certificación } \\
\text { código QR }\end{array}$ & $\begin{array}{l}\text { Generador QR } \\
\text { Plataforma } \\
\text { Moodle } \\
\text { Zoom }\end{array}$ \\
\hline
\end{tabular}

Al seleccionar la temática de estudio, el participante accede a la información, recursos, actividades y evaluación del tema, los cuales se encuentran estructurados en los tres bloques de la metodología PACIE descritos en la introducción. La aprobación del curso estuvo configurada con el cumplimiento del $80 \%$ de las tareas asignadas y la aprobación de la evaluación presencial final. Durante el desarrollo del curso, se realizaron prácticas de la labor tutorial mediante la técnica de solución de casos. A fin de medir el rendimiento del curso y evaluar los conocimientos, habilidades y actitudes desarrolladas por los participantes, se utilizó la observación y e-rúbricas para la evaluación. Al finalizar el curso se tomó un examen presencial a todos los participantes, mismo que fue construido en línea con 40 preguntas cerradas con relación a los contenidos de los siete módulos. En el módulo de graduación, adicionalmente se aplicó una encuesta de satisfacción tipo Likert de 23 preguntas, 21 de ellas comprendieron cuatro aspectos: 1) académico, 2) técnico y tecnológico, 3) desempeño docente 4) implementación del curso en línea; las 2 preguntas restantes fueron de tipo abiertas, con un enfoque cualitativo orientado a determinar las fortalezas y debilidades del curso. Los participantes podían seleccionar su grado de satisfacción entre 1 y 5 , siendo 5 el valor más alto de satisfacción. Es preciso recalcar que todos los instrumentos para la evaluación del aprendizaje y satisfacción de los participantes fueron validados por pares expertos de la Universidad Técnica del Norte.

\section{RESULTADOS}

Los resultados más relevantes se detallan en cuatro apartados: 1) diagnóstico sobre el nivel de competencias digitales, 2) rendimiento del curso, 3) implementación y 4) satisfacción del curso.

\section{Diagnóstico sobre el nivel de competencias digitales}

El 67,89\% de los asistentes al curso de Formación para Tutores Virtuales manifestó tener al menos un año de experiencia como docentes en la modalidad presencial. Así también, el 67,87\% expresó no tener experiencia como docentes virtuales, sin embargo, de este segmento, el 59,61\% afirmó haber cursado algún tipo de formación en línea. Del 32,13\% que afirmó tener experiencia como docentes virtuales, el 42\% indicó que su labor estuvo enmarcada dentro del área académica, $26 \%$ en el área administrativa y el $32 \%$ en el área técnico-pedagógica.

En cuanto a su experiencia en el uso de las TIC para el desarrollo del proceso de enseñanza-aprendizaje, el $62,23 \%$ afirmó usarlas de forma frecuente, el $26,77 \%$ de forma moderada y apenas el $5,36 \%$ no las usan por incomodidad y/o desconocimiento de su integración en el aula. Al preguntar si se encuentra capacitado para realizar la labor tutorial en educación en línea, el 52\% manifestó que puede hacerlo en un nivel avanzado. Por otro lado, el nivel de competencias digitales del primer grupo (Información y alfabetización informacional), el $54,32 \%$ dice conocer en un nivel avanzado cómo almacenar información en la nube 
(Google Drive, OneDrive, Dropbox), y el 41,34\% conoce como buscar información en Internet usando estrategias de navegación. Referente al segundo grupo (Comunicación y colaboración), el $52,38 \%$ conoce en un nivel avanzado como compartir información en la Red y un $32,7 \%$ dice conocer y utilizar redes sociales como Facebook, Twitter y/o YouTube. Asimismo, un 23,7\% indica que su nivel de conocimiento es básico referente a herramientas digitales como Zoom, Hangouts, Skype.

Con relación al tercer grupo (Creación de Contenido Digital), un 17,30\% expresa conocer en un nivel avanzado herramientas como Prezi, Emaze y Sway; el 54,31\% Office 365 y 2,40\% Kahoot como herramientas para gamificar el aprendizaje. En cuanto al cuarto componente (Seguridad), el $85,57 \%$ indica conocer en un nivel intermedio la seguridad de los datos, netiqueta e identidad digital, seguido por el $86,53 \%$ que afirma conocer conceptos y legislación sobre de derechos de autor y licencias de uso. Finalmente, para el quinto grupo (Resolución de problemas), el 49,03\% dice conocer en un nivel avanzado las configuraciones básicas de dispositivos digitales.

\section{Rendimiento del curso en línea}

Para medir el rendimiento del curso, para cada módulo se realizaron diferentes actividades y tareas, cada una con su respectiva e-rúbrica de evaluación. Con la escala de valoración establecida acorde a la actividad práctica, los participantes demostraron su aprendizaje y habilidad tutorial. Los resultados fueron altamente positivos; en la Tabla 5 se muestra una síntesis de las actividades con el resultado de la evaluación promedio para cada tarea. El promedio final de evaluación obtenido por los participantes fue de 8,78 .

Tabla 5. Rendimiento de los participantes en el curso

\begin{tabular}{|c|c|c|c|}
\hline Módulo & & Actividad & Promedio \\
\hline \multirow[t]{2}{*}{ Introductorio } & \multicolumn{2}{|r|}{ Moodle: Edición Perfil } & 8.48 \\
\hline & \multicolumn{2}{|c|}{$\begin{array}{l}\text { Flipgrid: Video reflexión estudiar en línea } \\
\text { (Resolución casos) }\end{array}$} & 8.56 \\
\hline \multirow[t]{2}{*}{ Tutor Virtual } & \multicolumn{2}{|c|}{ Presentación: Tutor virtual (Resolución casos) } & 8.73 \\
\hline & \multicolumn{2}{|c|}{$\begin{array}{l}\text { Matriz: Factores de la educación en línea-Labor tutorial } \\
\text { (Resolución casos) }\end{array}$} & 8.93 \\
\hline \multirow{2}{*}{\multicolumn{2}{|c|}{ Procesos Tutor Virtual }} & Casos: Resolución de casos tutoriales & 9.29 \\
\hline & & Documento: Acompañamiento al estudiante & 8.47 \\
\hline \multirow{2}{*}{\multicolumn{2}{|c|}{ Herramientas TIC }} & Glosario: Herramienta TIC & 9.29 \\
\hline & & $\begin{array}{l}\text { Zoom: Videoconferencia } \\
\text { (Resolución casos) }\end{array}$ & 9.34 \\
\hline \multicolumn{2}{|c|}{ Objetos virtuales de aprendizaje } & $\begin{array}{l}\text { OVA: Diseño } \\
\text { (Resolución casos) }\end{array}$ & 8.74 \\
\hline \multicolumn{2}{|c|}{ Examen final-presencial } & Cuestionario & 7.96 \\
\hline
\end{tabular}

\section{Implementación y satisfacción del curso}

Los resultados obtenidos al finalizar el curso virtual, demostraron que hubo un mejoramiento en cuanto a las competencias digitales de los participantes. Al preguntar si se encuentran capacitados para realizar la labor tutorial de educación en línea, el $87 \%$ manifestó que puede hacerlo en un nivel avanzado. En cuanto al mejoramiento de las competencias digitales en sus cinco componentes y de acuerdo con la percepción de los participantes, se pudo evidenciar un crecimiento importante en sus habilidades y destrezas para manejar las diferentes herramientas TIC en un nivel avanzado. Ver Tabla 6.

Tabla 6: Competencia digital inicial vs desarrollo de la competencia digital al finalizar el curso

\begin{tabular}{|l|c|c|c|c|}
\hline \multicolumn{1}{|c|}{ Componente competencia digital } & $\begin{array}{c}\text { Resultado } \\
\text { diagnóstico }\end{array}$ & $\begin{array}{c}\text { Promedio } \\
\text { competencia }\end{array}$ & $\begin{array}{c}\text { Resultado al } \\
\text { finalizar el curso }\end{array}$ & $\begin{array}{c}\text { Promedio } \\
\text { competencia }\end{array}$ \\
\hline Información y alfabetización informacional & $54.32 \%$ & \multirow{2}{|c|}{$47.83 \%$} & $91.34 \%$ & \multirow{2}{*}{$93.75 \%$} \\
\cline { 1 - 2 } $\begin{array}{l}\text { Almacenar la información en Google Drive, } \\
\text { OneDrive, Dropbox }\end{array}$ & & $96.15 \%$ & \\
\hline $\begin{array}{l}\text { Buscar información en Internet usando estrategias } \\
\text { de navegación }\end{array}$ & $41.34 \%$ & & \\
\hline
\end{tabular}


Tabla 6: continuación.

\begin{tabular}{|c|c|c|c|c|}
\hline \multicolumn{5}{|l|}{ Comunicación y colaboración } \\
\hline Compartir información en la Red & $52.38 \%$ & \multirow{3}{*}{$42.54 \%$} & $92.79 \%$ & \multirow{3}{*}{$91.66 \%$} \\
\hline Redes sociales Facebook, Twitter y YouTube & $32.70 \%$ & & $95.67 \%$ & \\
\hline Zoom, Hangouts, Skype & $42.53 \%$ & & $86.53 \%$ & \\
\hline \multicolumn{5}{|l|}{ Creación de Contenido Digital } \\
\hline Prezi, Emaze, Sway & $17.30 \%$ & \multirow{3}{*}{$24.67 \%$} & $89.90 \%$ & \multirow{3}{*}{$90.86 \%$} \\
\hline Office 365 & $54.31 \%$ & & $85.57 \%$ & \\
\hline Kahoot & $2.40 \%$ & & $97.11 \%$ & \\
\hline \multicolumn{5}{|l|}{ Seguridad } \\
\hline $\begin{array}{l}\text { Seguridad de los datos, netiqueta e identidad } \\
\text { digital }\end{array}$ & $3.5 \%$ & \multirow[t]{2}{*}{$2.92 \%$} & $85.09 \%$ & \multirow[t]{2}{*}{$84.37 \%$} \\
\hline Derechos de autor y licencias de uso & $2.33 \%$ & & $83.65 \%$ & \\
\hline \multicolumn{5}{|l|}{ Resolución de problemas } \\
\hline Configuraciones básicas de dispositivos digitales & $49.03 \%$ & $49.03 \%$ & $76.44 \%$ & $76.44 \%$ \\
\hline
\end{tabular}

Finalmente, al terminar el curso se realizó una encuesta a todos los participantes para conocer su opinión respecto al curso virtual, a fin de obtener la retroalimentación que permita corroborar los resultados y corregir las falencias que puedan existir. La encuesta fue contesta por el 69,71\% (145 participantes) y se logró evidenciar su satisfacción de forma positiva en el ámbito académico, técnico-tecnológico, desempeño docente y la implementación del curso en línea. Ver Tabla 7.

Tabla 7: Valoración de la Satisfacción del curso. VM es valoración máxima

\begin{tabular}{|c|c|}
\hline Aspectos de medición & $\% V M(5)$ \\
\hline \multicolumn{2}{|l|}{ Aspectos Académicos } \\
\hline Se logró cumplir los objetivos planificados al inicio del curso. & $100 \%$ \\
\hline $\begin{array}{l}\text { El curso le permitió el desarrollo de nuevas experiencias, competencias y habilidades para } \\
\text { desenvolverse en el proceso de formación en línea. }\end{array}$ & $98 \%$ \\
\hline $\begin{array}{l}\text { Las actividades realizadas en cada módulo le permitieron poner en práctica su aprendizaje y } \\
\text { competencias adquiridas en el curso. }\end{array}$ & $98 \%$ \\
\hline Las evaluaciones aplicadas fueron adecuadas con relación a los contenidos del curso. & $99 \%$ \\
\hline El nivel de exigencia del examen final fue adecuado & $97 \%$ \\
\hline \multicolumn{2}{|l|}{ Aspectos técnico y tecnológicos } \\
\hline Los recursos didácticos utilizados durante el curso facilitaron su aprendizaje. & $97 \%$ \\
\hline Los contenidos de cada módulo fueron reforzados a través de foros y videoconferencia. & $98 \%$ \\
\hline Los tiempos establecidos para el desarrollo de las actividades fue adecuado & $91 \%$ \\
\hline Se cumplió con los tiempos y horarios establecidos para cada actividad & $100 \%$ \\
\hline $\begin{array}{l}\text { La herramienta Zoom permitió el desarrollo satisfactorio de las clases presenciales virtuales } \\
\text { (videoconferencia) }\end{array}$ & $99 \%$ \\
\hline \multicolumn{2}{|l|}{ Aspectos del desempeño docente } \\
\hline El docente tutor demostró conocimiento y domino en los contenidos impartidos. & $100 \%$ \\
\hline El docente tutor estimuló su aprendizaje y participación durante el curso. & $100 \%$ \\
\hline El docente tutor propició un espacio de crítica y reflexión entre los participantes. & $98 \%$ \\
\hline La retroalimentación o respuesta a sus inquietudes fue oportuna. & $100 \%$ \\
\hline El lenguaje utilizado por el docente tutor fue apropiado & $100 \%$ \\
\hline Aspectos de medición & $\% V M(5)$ \\
\hline \multicolumn{2}{|l|}{ Aspectos de la implementación del curso } \\
\hline $\begin{array}{l}\text { Las instrucciones ofrecidas para el manejo de la plataforma virtual fueron claras y fáciles de poner en } \\
\text { práctica. }\end{array}$ & $97 \%$ \\
\hline La metodología de enseñanza en línea (PACIE) aplicada en el curso para su formación fue apropiada. & $98 \%$ \\
\hline La estructura del curso en Moodle le permitió tener una experiencia de aprendizaje satisfactoria & $98 \%$ \\
\hline $\begin{array}{l}\text { Los contenidos fueron relevantes para mejorar su competencia digital y desenvolverse como tutor } \\
\text { virtual. }\end{array}$ & $98 \%$ \\
\hline Está satisfecho con el nivel de conocimientos y habilidades alcanzadas en el curso & $96 \%$ \\
\hline Su participación en el curso fue activa & $97 \%$ \\
\hline
\end{tabular}

De la misma manera se puede apreciar en la Tabla 8 una síntesis de las respuestas recogidas en las dos preguntas abiertas, las mismas que ponen en manifiesto su valoración significativa en cuanto a lo aprendido y a la mejora de sus competencias digitales. 
Tabla 8: Preguntas abiertas.

\begin{tabular}{|l|l|}
\hline Pregunta & Respuesta consolidada \\
\hline $\begin{array}{l}\text { ¿Qué es lo que } \\
\text { más le gustó del } \\
\text { curso en línea? }\end{array}$ & $\begin{array}{l}\text { Los cursos virtuales deben ser orientados de esta manera. } \\
\text { Las prácticas fueron interesantes porque pudimos aplicar lo aprendido y corregir nuestros } \\
\text { erroren la retroalimentación del profesor. }\end{array}$ \\
& $\begin{array}{l}\text { Herramientas muy valiosas para el ejercicio profesional. } \\
\text { La predisposición del tutor para contestar nuestras inquietudes. } \\
\text { Las videoconferencias } \\
\text { Mejoré mi competencia digital } \\
\text { Incorporar las TIC para promover el aprendizaje de forma lúdica. }\end{array}$ \\
\hline $\begin{array}{l}\text { ¿Qué nos sugiere } \\
\text { mejorar en este } \\
\text { curso? }\end{array}$ & $\begin{array}{l}\text { Ampliar el tiempo para realizar las actividades. } \\
\text { El curso debe ser más pausado, debido a que no manejamos correctamente estos sistemas. } \\
\text { Ampliar el tiempo para profundizar más las herramientas TIC. }\end{array}$ \\
& $\begin{array}{l}\text { La práctica del rol de tutor virtual debe ser con la participación del tutor porque algunos } \\
\text { compañeros no evaluaron de forma profesional la misma. } \\
\text { Bajar el nivel del examen final o la ponderación. }\end{array}$ \\
\hline
\end{tabular}

\section{DISCUSIÓN}

Esta investigación, se enfocó en la importancia y relación de las competencias digitales con la calidad de la educación virtual; sin embargo, también se debe recalcar el aporte en los ámbitos organizacional, académico, pedagógico y técnico que representa la implementación de una metodología práctica para la formación de tutores virtuales. Los resultados obtenidos en este estudio de caso ponen en evidencia las falencias en cuanto a competencias digitales de los docentes universitarios en el Ecuador, las cuales se ven maximizadas cuando hacemos referencia a modelos educativos online. De acuerdo con los resultados de Bravo et al., (2019), las competencias digitales deben ser parte de la formación tecno-pedagógica de los tutores virtuales, permitiendo la integración de las TIC a los procesos educativos y edificando una perspectiva constructivista que de soporte a los retos actuales y futuros (Centeno y Cubo, 2013).

Por otra parte, González et al., (2012) afirman que el docente limita el desarrollo de las competencias digitales de sus estudiantes, si éste no tiene un dominio avanzado de dichas competencias. Es inaceptable que, en la era digital, la falta de conocimiento y confianza en el uso de las TIC, causen incomodidad e impidan que el docente adopte nuevos enfoques tecno-pedagógicos (Maderick et al., 2016). Es necesario que el docente comprenda la importancia de las e-skills dentro del proceso de formación en línea, a fin de que pueda incidir positivamente en los grupos de estudiantes que presenten menores capacidades dentro de este contexto.

Conforme se iba avanzando en la ejecución del curso virtual, los participantes fueron alcanzando un mayor nivel de experticia digital, familiarizándose con el uso de espacios virtuales y desarrollando una sensibilidad pedagógica y humanística que a la postre les permitirá acompañar a sus estudiantes en el proceso de adaptación, desarrollo y formación. La evaluación final confirmó una mejora significativa en las competencias digitales de los asistentes al curso, corroborando los datos obtenidos por Thompson (2013). Según la percepción de los participantes, el grupo de competencias predominante es el de información y alfabetización informacional y el de comunicación y colaboración, datos que tienen similitud con los resultados de la investigación realizada por Rolf et al., (2019).

El adecuado diagnóstico de las competencias digitales de los asistentes al curso para "Formación de Tutores Virtuales", permitió planificar los contenidos a la medida de los estudiantes y establecer el proceso de seguimiento y evaluación a fin de determinar el nivel de mejora en el aprendizaje y garantizar la transferencia de conocimientos y herramientas requeridas por los mismos; esto ratifica lo hallado por Caena y Redecker (2019) y Morán et al., (2015) donde el proceso de diagnóstico y la reflexión del nivel de la competencia de los docentes propicia una comprensión clara de las necesidades de aprendizaje profesional que facilita incorporar estrategias tecnopedagógicas necesarias para impulsar la innovación en la educación del siglo XXI.

En lo referente a la implementación y el grado de satisfacción del curso en línea, la encuesta presentó una relativa inconformidad de los estudiantes relacionado con el tiempo destinado para cada actividad y la rigurosidad del tutor a la hora de valorar el nivel competencias digitales. La limitada literatura científica existente sobre el tema investigado, impide que se haga un análisis y contraste más amplio; sin embargo, el criterio de nivel profesional es clave para los objetivos de la investigación ya que su retroalimentación permitirá realizar futuras mejoras a este proceso formativo. Al no estratificar la población que contestó la encuesta por dos elementos: nivel de experiencia en la modalidad online y título de formación académica que posee, se limita conocer con certeza las diferencias en los resultados de quienes poseen estos elementos de quienes no. 
Sin embargo, las limitaciones de los participantes sirve para retroalimentar y reforzar el macro-proceso de formación online de acuerdo con los siguientes aspectos: 1) impulsar el cambio cultural del proceso de enseñanza-aprendizaje de esta modalidad en el docente tutor y aprendices, 2) dar mayor valoración o peso al aprendizaje colaborativo e interactivo como vías prioritarias en el desarrollo competencial, 3) revisar el número de actividades de aprendizaje en consideración con los plazos de entrega, 4) realizar un análisis de las estrategias didácticas empleadas en la formación online a fin de determinar su eficacia en el aprendizaje de los futuros tutores virtuales, 5) es necesario evaluar de forma continua la formación en competencia digitales y la medición de su eficacia como herramienta para el desarrollo docente.

No obstante, también se pudo evidenciar la satisfacción en referencia a la transición de los conocimientos teóricos a la práctica. Por ello, es indispensable que la formación de tutores virtuales se realice bajo modalidad virtual, generando una experticia digital, desarrollando las habilidades, conocimientos, actitudes y estrategias para el uso eficiente y seguro de las TIC. En consonancia a lo encontrado por Sjølie (2014), las competencias digitales como parte de la formación de los docentes no es un deseo, sino una necesidad crítica para alcanzar la calidad en el entorno educativo universitario.

\section{CONCLUSIONES}

De acuerdo a los resultados y discusión de este estudio, se logró establecer las siguientes conclusiones: 1) la implementación del curso online basado en la metodología PACIE fue exitoso para la formación de tutores virtuales ya que conjuga la teoría de las TIC con la aplicación metodológica de las mismas; 2) la formación de tutores virtuales en competencias digitales permite ampliar la cobertura de educación utilizando las TIC como herramienta de enseñanza, aplicando en esta, la pedagogía, andragogía y más elementos psicopedagógicos indispensables en el proceso de aprendizaje, enseñanza-aprendizaje o interaprendizaje; 3 ) el estudio demuestra cuán importante es en la globalización de la enseñanza las competencias digitales fusionadas al quehacer metodológico de la labor docente, lo que se visibiliza en el desarrollo de habilidades, conocimientos, actitudes y estrategias con el uso eficiente y seguro de las tecnologías de información y comunicación en educación en línea.

\section{AGRADECIMIENTOS}

Los autores agradecen a la Universidad Técnica del Norte por la apertura y el apoyo para el desarrollo de esta investigación que forma parte de una tesis doctoral, realizada dentro del Programa de Doctorado "Education in the Knowledge Society", de la Universidad de Salamanca (España).

\section{REFERENCIAS}

Basantes-Andrade, A., Cabezas-González, M., y Casillas-Martín, S., Digital competences relationship between gender and generation of university professors. https://doi.org/10.18517/ijaseit.10.1.10806, International Journal on Advanced Science, Engineering and Information Technology, 10(1), 205-211 (2020)

Basantes-Andrade, A., Cabezas-González, M., y Casillas-Martín, S., Digital competences in e-learning. Case study: Ecuador. https://doi.org/10.1007/978-3-030-37221-7_8, In International Conference on Knowledge Society: Technology, Sustainability and Educational Innovation, 85-94 (2019).

Basantes, A. V., Naranjo, M, E., y Ojeda, V., Metodología PACIE en la educación virtual: una experiencia en la Universidad Técnica del Norte. https://doi.org/10.4067/S0718-50062018000200035, Form. Univ, 11(2), 35-44 (2018)

Basantes, A. V., Naranjo, M. E., Gallegos, M. C., y Benítez, N. M., Los dispositivos móviles en el proceso de aprendizaje de la Facultad de Educación Ciencia y Tecnología de la Universidad Técnica del Norte de Ecuador. https://doi.org/10.4067/S0718-50062017000200009, Form. Univ, 10(2), 79-88 (2017)

Bravo, A. A., Faúndez, C. A., Moraga, F. A., y Borzone, M. A., Formación de estudiantes tutores: un apoyo fundamental para potenciar el desarrollo de prácticas de física. https://doi.org/10.4067/S0718-50062017000200009, Form. Univ, 12(2), 63-72 (2019)

Caena, F., y Redecker, C., Aligning teacher competence frameworks to 21st century challenges: the case for the european digital competence framework for educators (Digcompedu). https://doi.org/10.4067/S07185006201800020003510.1111/ejed.12345, European Journal of Education, 54, 356-369 (2019)

Cabero, J., y Martínez, A., Las tecnologías de la información y comunicación y la formación inicial de los docentes: modelos y competencias digitales. https://doi.org/10.30827/profesorado.v23i3.9421, Profesorado, 23(3), 247-268 (2019)

Cazco, G. H., González, M. C., Abad, F. M., y Mercado-Varela, M. A., Digital competence of the university faculty: case study of the Universidad Nacional de Chimborazo. https://doi.org/10.1145/3012430.3012510, In Proceedings of the fourth international conference on technological ecosystems for enhancing multiculturality, 147-154 (2016)

Centeno, G. y Cubo, S, Evaluación de la competencia digital y las actitudes hacia las TIC del alumnado universitario. https://doi.org/10.6018/rie.31.2.169271, Revista de Investigación Educativa, 31(2), 517-536 (2013) 
Garzón, E., Martínez, T., y otros tres autores, Teacher Training in Lifelong Learning - The Importance of Digital Competence in the Encouragement of Teaching Innovation. https://doi.org/10.3390/su12072852, Sustainability, 12(7), 1$13(2020)$

González, J., Espuny, C., de Cid, M. J. y Gisbert, M., INCOTIC-ESO. Cómo autoevaluar y diagnosticar la competencia digital en la escuela 2.0. https://doi.org/10.6018/rie.30.2.117941, Revista de Investigación Educativa, 30 (2), $287-302$ (2012)

INTEF, Marco común de competencia digital docente, $2^{\text {da }}$ edición, 1-69. INTEF, Madrid, España (2017)

Janssen, J., Stoyanov, S, y otros cuatro autores, Experts' views on digital competence: commonalities and differences. https://doi.org/10.1016/j.compedu.2013.06.008, Computers y Education, 68(1), 473-481 (2013)

Llorente, M., El tutor en e-learning: aspectos a tener en cuenta. https://doi.org/10.21556/edutec.2006.20.517, Revista Electrónica de Tecnología Educativa, 1(20), 1-16 (2006)

Maderick, J. A., Zhang, S., Hartley, K., y Marchand, G., Preservice teachers and self-assessing digital competence. https://doi.org/10.1177/0735633115620432, Journal of Educational Computing Research, 54(3), 326-351 (2016)

Martínez, O., Steffens, E., Ojeda, C., y Hernández, H., Estrategias pedagógicas aplicadas a la educación con mediación virtual para la generación del conocimiento global. https://doi.org/10.4067/S0718-50062018000500011, Form. Univ, 11(5), 11-18 (2018)

McDougall, J., 'I never felt like I was alone': a holistic approach to supporting students in an online, pre-university programme. Open Learning: The Journal of Open. https://doi.org/10.1080/02680513.2019.1583098, Distance and eLearning, 34(3), 241-256 (2019)

Morán, R., Cardoso, E., Cerecedo, M., y Ortíz, J., Evaluación de las competencias docentes de profesores formados en instituciones de educación superior: el caso de la asignatura de tecnología en la enseñanza secundaria. https://doi.org/10.4067/S0718-50062015000300007, Form. Univ, 8(3), 57-64 (2015)

Nyikes, Z., Digital competence and the safety awareness base on the assessments results of the middle east-european generations, Procedia Manufacturing, ISSN: 23519789, 22(1), 916-922, (2018)

Oses, S., y Carrasco, L., Módulos alternativos en la enseñanza de las ciencias: estrategia didáctica orientada al logro de aprendizajes significativos. https://doi.org/10.4067/S0718-50062013000300006, Form. Univ, 6(3), $39-52$ (2013)

Pérez, A. y Rodríguez, M. J., Evaluación de las competencias digitales autopercibidas del profesorado de educación primaria en Castilla y León. https://doi.org/10.6018/rie.34.2.215121, Revista de Investigación Educativa, 34(2), 399-415 (2016)

Prendes, M, Gutiérrez, I., y Martínez, F., Competencia digital: una necesidad del profesorado universitario en el siglo XXI. https://doi.org/10.6018/red/56/7, RED: Revista de Educación a Distancia, 56, 1-22 (2018)

Rodrigues, H., Almeida, F., Figueiredo, V., y Lopes, S. L., Tracking e-learning through published papers: A systematic review. https://doi.org/10.1016/j.compedu.2019.03.007, Computers y Education, 136(1), 87-98 (2019)

Røkenes, F., y Krumsvik, R., Prepared to teach ESL with ICT? A study of digital competence in norwegian teacher education. https://doi.org/10.1016/j.compedu.2016.02.014, Computers y Education, 97, 1-20 (2016)

Rolf, E., Knutsson, O., y Ramberg, R., An analysis of digital competence as expressed in design patterns for technology use in teaching. https://doi.org/10.1111/bjet.12739, British Journal of Educational Technology, 50(6), 3361-3375 (2019)

Senescyt, El programa de educación superior virtual impulsado por la senescyt ofrecerá 30.000 nuevos cupos, (en la web: http://cort.as/-MTLd, acceso: 11 de Diciembre 2019, CEAACES, Quito (2018)

Sjølie, E., The role of theory in teacher education: reconsidered from a student teacher perspective. https://doi.org/10.1080/00220272.2013.871754, Journal of Curriculum Studies, 46(6), 729-750 (2014)

Tømte, C., Enochsson, A. B., Buskqvist, U., y Kårstein, A., Educating online student teachers to master professional digital competence: the tpack-framework goes online. https://doi.org/10.1016/j.compedu.2015.01.005, Computers y Education, 84, 26-35 (2015)

Thompson, P., The digital natives as learners: technology use patterns and approaches to learning. https://doi.org/10.1016/j.compedu.2012.12.022, Computers y Education, 65, 12-33 (2013)

Villarreal-Villa, S., García-Guliany, J., Hernández-Palma, H., y Steffens-Sanabria, E., Competencias docentes y transformaciones en la educación en la era digital. https://doi.org/10.4067/S0718-50062019000600003, Form. Univ, 12(6), 3-14 (2019) 
